\title{
Hardware Development for the Radio Neutrino Observatory in Greenland (RNO-G)
}

\author{
Daniel Smith $^{a, *}$ on behalf of The RNO-G Collaboration \\ (a complete list of authors can be found at the end of the proceedings) \\ ${ }^{a}$ University of Chicago, Department of Physics, 5720 S Ellis Ave, Chicago, IL 60637, USA \\ E-mail: danielsmith@uchicago.edu
}

The Radio Neutrino Observatory in Greenland (RNO-G) is designed to make the first observations of ultra-high energy neutrinos at energies above $10 \mathrm{PeV}$, playing a unique role in multi-messenger astrophysics as the world's largest in-ice Askaryan radio detection array. The experiment will be composed of 35 autonomous stations deployed over a 5 x $6 \mathrm{~km}$ grid near NSF Summit Station in Greenland. The electronics chain of each station is optimized for sensitivity and low power, incorporating $150-600 \mathrm{MHz} \mathrm{RF}$ antennas at both the surface and in ice boreholes, low-noise amplifiers, custom RF-over-fiber systems, and an FPGA-based phased array trigger. Each station will consume $25 \mathrm{~W}$ of power, allowing for a live time of $70 \%$ from a solar power system. The communications system is composed of a high-bandwidth LTE network and an ultra-low power LoRaWAN network. I will also present on the calibration and DAQ systems, as well as status of the first deployment of 10 stations in Summer 2021.

${ }^{*}$ Presenter

$37^{\text {th }}$ International Cosmic Ray Conference (ICRC 2021)

July 12th - 23rd, 2021

Online - Berlin, Germany 


\section{Radio Neutrino Observatory in Greenland}

The Radio Neutrino Observatory in Greenland (RNO-G) is an in-ice Askaryan radio detection array near to NSF Summit Station [1]. RNO-G is currently under construction, with the first stations being deployed to Greenland in Summer 2021. By the end of 2023, RNO-G will be the world's largest in-ice radio detector and, after a five year science run, will have a world-leading sensitive to the diffuse astrophysics neutrino flux at energies above $10 \mathrm{PeV}$. For a more complete description of the detector and its science goals, please see the detector paper in Citation [1].

RNO-G is designed to detect the first astrophysics neutrinos at energies above $10 \mathrm{PeV}$. RNO-G will extend the energy range of the IceCube Collaboration's already observed astrophysical neutrino flux [2]. Through the detection of astrophysical neutrinos, RNO-G will participate in multimessenger astronomy, a new paradigm of astronomy where multiple observed particles species are used to study astrophysical sources in greater detail than possible before. IceCube's recent promising correlations of neutrino events with a blazar [3] and tidal disruption event [4] show that there is much potential for astrophysical neutrino multimessenegery astronomy. Besides extending IceCube's sensitivity, RNO-G complements IceCube in sky coverage: RNO-G will cover the null in sensitivity created by the Earth in IceCube's sky coverage at ultra-high energies.

Besides measuring astrophysical neutrinos, RNO-G will also make cosmological measurements through the detection or non-observation of cosmogenic neutrinos (neutrinos made from the interaction of cosmic rays with photons in the cosmic microwave background), making independent measurements of cosmic ray composition and information on the source evolution of our universe $[1,5]$.

RNO-G will be composed of 35 autonomous stations in a $5 \times 6 \mathrm{~km}$ grid. Each station is composed of the following:

- Data acquisition system (DAQ) inside of an environmental enclosure, composed of:

- In-house developed, 24 channel digitizer board that utilizes the LAB4D ASIC [6]

- 4-channel, low threshold trigger board with FPGA-based phased array trigger [7]

- Controller board powered by a commercial single board computer

- Low-noise amplifiers for receiver of surface antennas and RF-over-fiber receivers for deep antennas

- GPS receiver for timing and position calibration

- Calibration pulser source with transmitter to 'helper strings'

- Deep science antennas deployed $100 \mathrm{~m}$ down into the ice in 11.2" diameter boreholes. The equipment down each borehole, called 'strings', are:

- 1x 'power string' with four vertically polarized antennas for phased array triggering

$-2 \mathrm{x}$ 'helper strings' for azimuth reconstruction of events and calibration

- Surface science antennas used for surface air shower tagging, noise rejection and improved reconstruction of deep-triggered events

- Power system of solar panels, a battery bank and the future possible addition of a wind turbine

- Wireless communication via an LTE and LoRAWAN network 


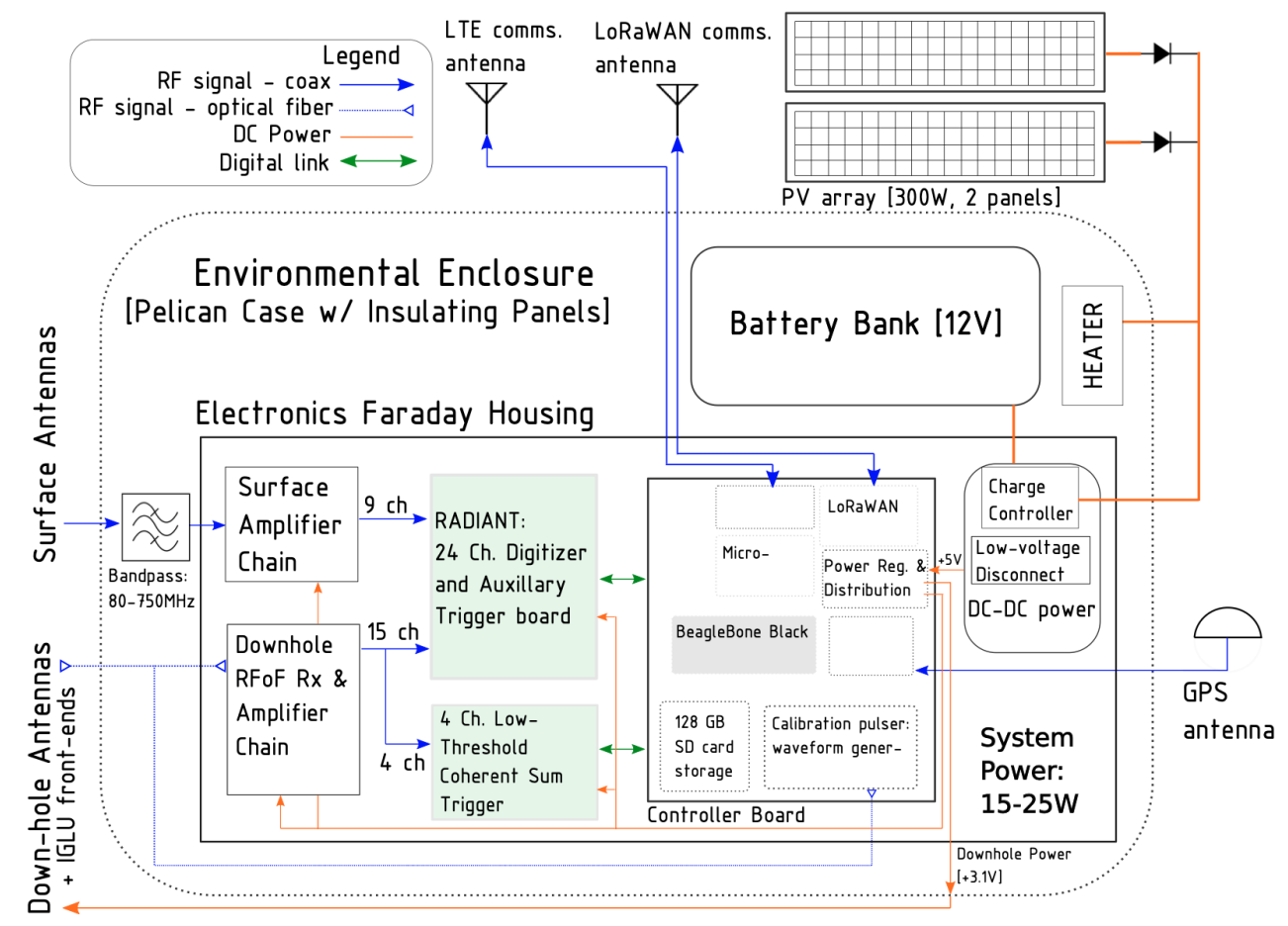

Figure 1: System diagram of a single RNO-G station, including all components described in the text.

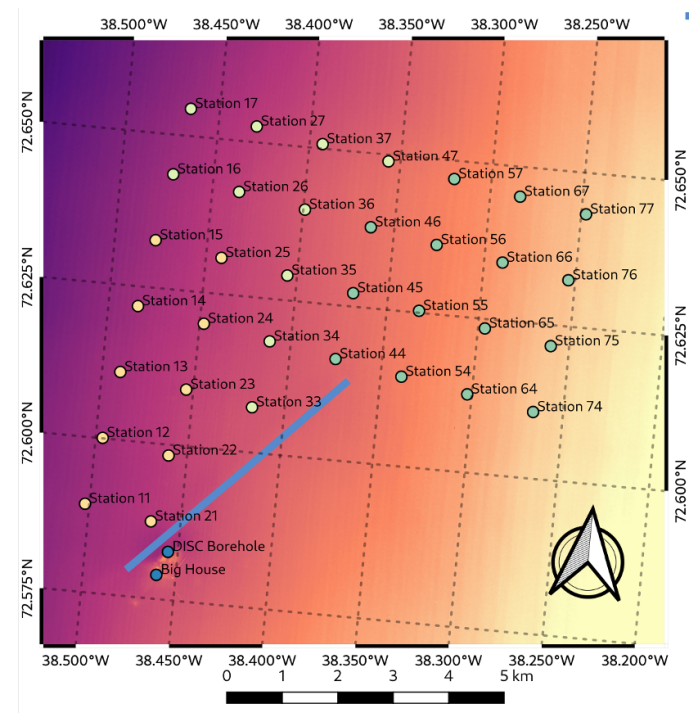

Figure 2: Top: Planned layout of the 35 stations in RNO-G and their position relative to Summit Station (marked by blue line of the landing strip). Background scale denotes altitude of $3240 \mathrm{~m}$ for purple and $3260 \mathrm{~m}$ for yellow.

Right: Layout of science antennas of a single station, including the power string and helper strings down boreholes in ice.

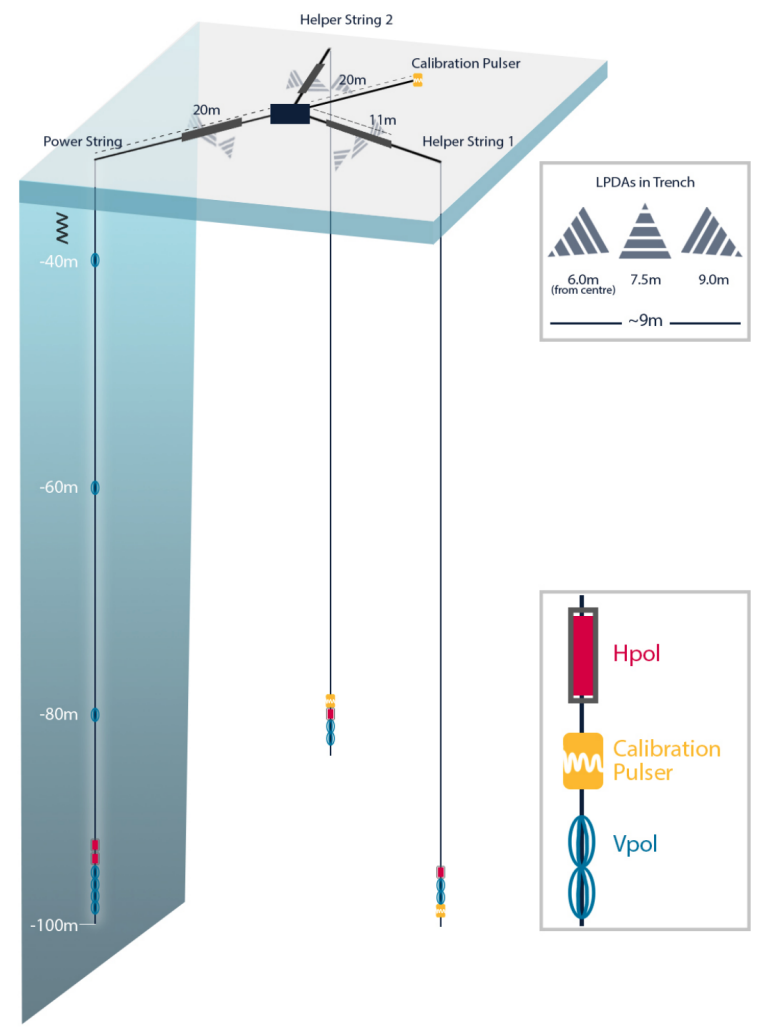




\section{RF Signal Chain}

The radio frequency (RF) signal chain takes two forms, one each for the deep and shallow antennas. For shallow antennas, RF is fed over $\sim 20 \mathrm{~m}$ coaxial cables to custom, low-power and low-noise amplifiers (LNAs) on the 'SURFACE' board that are deployed inside of the DAQ environmental enclosure. The signals are amplified by $\sim 60 \mathrm{dBm}$ before being fed into the digitizer and surface trigger on the 'RADIANT' board. For deep antennas, RF is fed directly into an LNA and RF-over-fiber optical transmitter $(\mathrm{RFoF})$ that is co-deployed in the borehole with each antenna. The now optical signals travel $\sim 120 \mathrm{~m}$ in fiber before entering an optical receiver. The RFoF receiver, within the DAQ environmental enclosure on the 'DRAB' board, converts the signal back to analog, amplifies it again before inserting the signal into the digitizer board and low-threshold triggering 'FLOWER' board. RF from the deep component has a total of $\sim 60 \mathrm{dBm}$ amplification before the digitizer.

The RFoF system removes the need for deploying coaxial cables down to each antenna, which minimizes weight and amount of dielectric material running through upper antennas while minimizing noise temperature $(<150 \mathrm{~K})$. The front-end LNA and RFoF system on the 'IGLU' boards were developed in-house to optimize for noise figure and power, with the final design consuming $140 \mathrm{~mW}$ per channel.

\section{Power and Communication Systems}

The detector will be composed of 35 autonomous stations deployed in a $5 \times 6 \mathrm{~km}$ grid. Each station must operate autonomously: Summit Station infrastructure is not equipped to handle cabling each station together for power and data transfer. For power, each station is equipped with two solar panels with a total power output of $300 \mathrm{~W}$ and a $5 \mathrm{kWh}$ sealed lead-acid battery bank. A wind power system is being developed to produce power for the stations during the winter months and may be deployed in a future season. Wireless communication is performed using two systems: a LoRAWAN and LTE network. LoRAWAN is an ultra-low power system for slow control and housekeeping data during the winter season. Commercial LTE offers high data bandwidth over large distances at the expense of power and is used for data telemetry to Summit Station.

Due to the lack of sun during arctic winter and before wind turbine deployment, stations will be left in a minimum low-power mode during the winter ( $30 \%$ of the year), with the only system online being an on-board micro-controller sending housekeeping data over LoRAWAN. The time before and after the winter are called 'shoulder seasons.' With minimum solar power, the stations will be set to a low-power mode where thermal trigger rates and data transfer are minimized to extend the productive up-time of the station. During the arctic summer, each station produces surplus power and can operate at high thermal trigger rates $(10 \mathrm{~Hz})$ and can operate over LTE continuously. During this operation mode, each station has a power budget of $\sim 25 \mathrm{~W}$. 

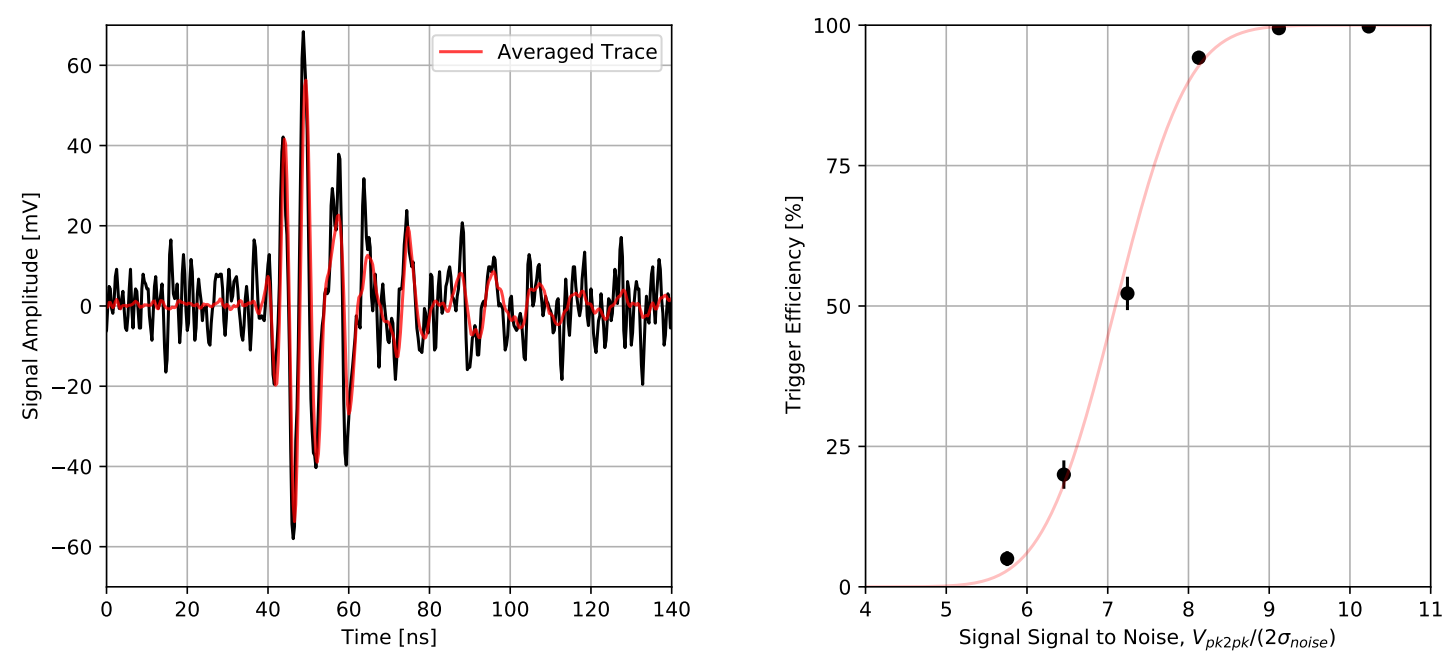

Figure 3: Left: Digitized trace of an $\mathrm{SNR}=9,100-225 \mathrm{MHz}$ band-passed impulse passed through the surface amplifiers. Red is the average trace, the source before the addition of thermal noise. Right: Preliminary trigger efficiency vs. SNR for the surface / air-shower trigger. Measurement taking in lab and before deployment. The trigger level results in a thermal noise trigger rate of $<1 \mathrm{~Hz}$. The trigger for the deep component is expected to have a 50\% trigger efficiency rate of $\sim 3.5 \mathrm{SNR}$.

\section{Digitizer}

Due to the specific power constraints of RNO-G, digitization of antenna signals is performed on an in-house developed board, the RAdio DIgitizer and Auxiliary Neutrino Trigger (RADIANT) board. The RADIANT is a 24 channel board that uses the LAB4D, a custom ASIC that is a single channel, switched-capacitor array with 12-bit sampling [6]. For the purposes of RNO-G, the LAB4Ds are operated at $3.2 \mathrm{GSa} / \mathrm{s}$ with two buffers of 2048 samples each, giving a total $1280 \mathrm{~ns}$ recorded window per channel. An example digitized trace from a generated fast impulse passed through the signal chain can be see in Figure 3.

\section{Trigger}

Each channel on the digitizer board has a low-power, Schottky diode detector circuit. The enveloped signal produced by the Schottky diode is compared between a DC voltage level and fed to the on-board FPGA to build a combinatoric trigger. The final combinatoric trigger will have a higher overall threshold than the primary science trigger, a phased array trigger, and will be used for surface antennas as an air shower trigger. A preliminary trigger efficiency vs. SNR can be seen in Figure 3.

The primary science trigger for each station will occur on the low-threshold trigger board, the so-called 'FLOWER' board. The low-threshold board is a 4-channel board with its own streaming, 8-bit, $500 \mathrm{MSa} / \mathrm{s}$ digitizers that feed into an FPGA. The FPGA will calculate, in real time, the expected delays between four deep, Vpol antennas for incident plane waves of various arrival 
directions, subtract the delays from the channels and sum the channels. This technique amplifies signal by $n_{\text {ant }}$ while amplifying noise by $\sqrt{n_{\text {ant }}}$, increasing the single-channel signal-to-noise ratio. RNO-G's trigger design is a lower-power version of that deployed in ARA [7]. By operating with four channels and using a $500 \mathrm{MSa} / \mathrm{s}$ digitizer, the trigger board is able to operate at $\sim 2.4 \mathrm{~W}$ while reaching a 50\% trigger efficiency of 3-4 SNR and maintaining a $\mathrm{O}(10 \mathrm{~Hz})$ thermal noise trigger rate. The phased array trigger technique has proven to greatly improve low SNR triggering and thus increasing detector volume [7].

\section{Science Antenna Development}

Each station is deployed with deep and shallow science antennas, each with their own design constraints, science goals and trigger configuration.

The shallow antennas are commercially available, high-gain, logperiodic dipole antennas (LPDAs). These antennas are deployed in slots in the snow that are back-filled, directly coupling the antennas to the snow. The surface antennas are large, broadband, have high resolution polarization reconstruction ability [8] and have been extensively calibrated by the ARIANNA collaboration [9]. Surface antennas will be used primar-

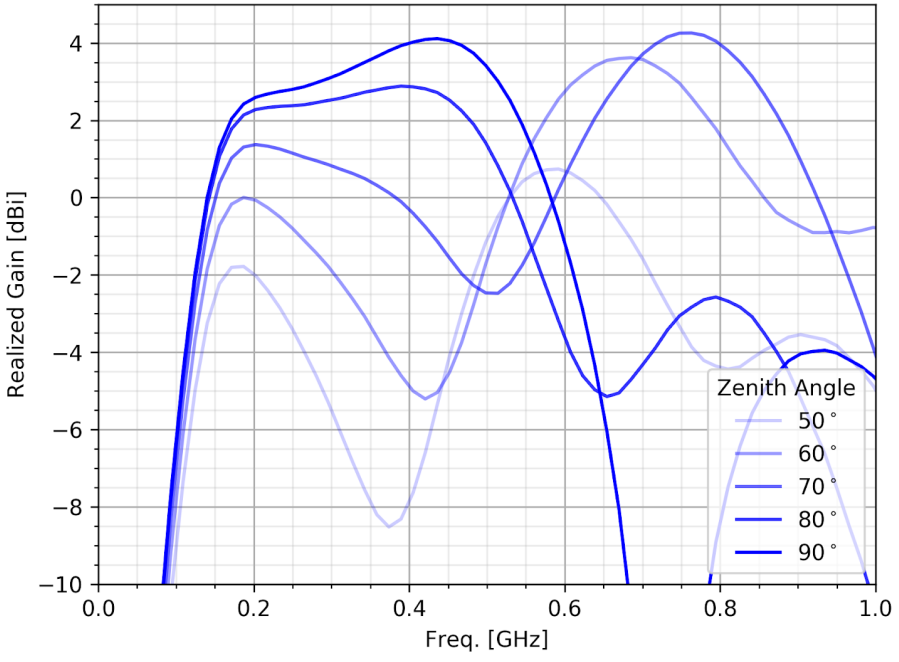

Figure 4: Simulated Realized Gain of the VPol antenna in $n=$ 1.75 ice inside of an 11.2" diameter air borehole. ily for air-shower tagging, noise rejection and improved event reconstruction for deep-triggered events.

The deep antennas are the primary sensing component of RNO-G. Deep antennas are deployed $100 \mathrm{~m}$ into the ice to avoid the loss of effective detector volume caused by the gradient of index of refraction in the top layers (firn) of Greenlandic ice [10,11]. Antenna design is a challenge due to the constrained geometry of the 11.2" diameter ice borehole. Simulation is a challenge due to the air / ice interface created by the borehole that exists in the near-field of the antenna.

The vertically polarized ( $\mathrm{Vpol}$ ) antenna takes the form of a fat-dipole antenna, iterating on the GNO and RICE antenna designs [12]. The design is sensitive in the band $150 \mathrm{MHz}-600$ Mhz and has a VSWR $<3$ over its entire band. The antenna is $60 \mathrm{~cm}$ tall, 5 " in diameter, and constructed in stock aluminum to reduce material and machining costs. The opening and design of the antenna feed was optimized for $50 \Omega$ matching in the time-domain xFDTD software package. In-situ measurements of the antenna response are ongoing using in-situ calibration pulsers as well as calibration campaigns made by mobile pulsers (on ei snowmobiles) and deep pulsers down the DISC borehole. 

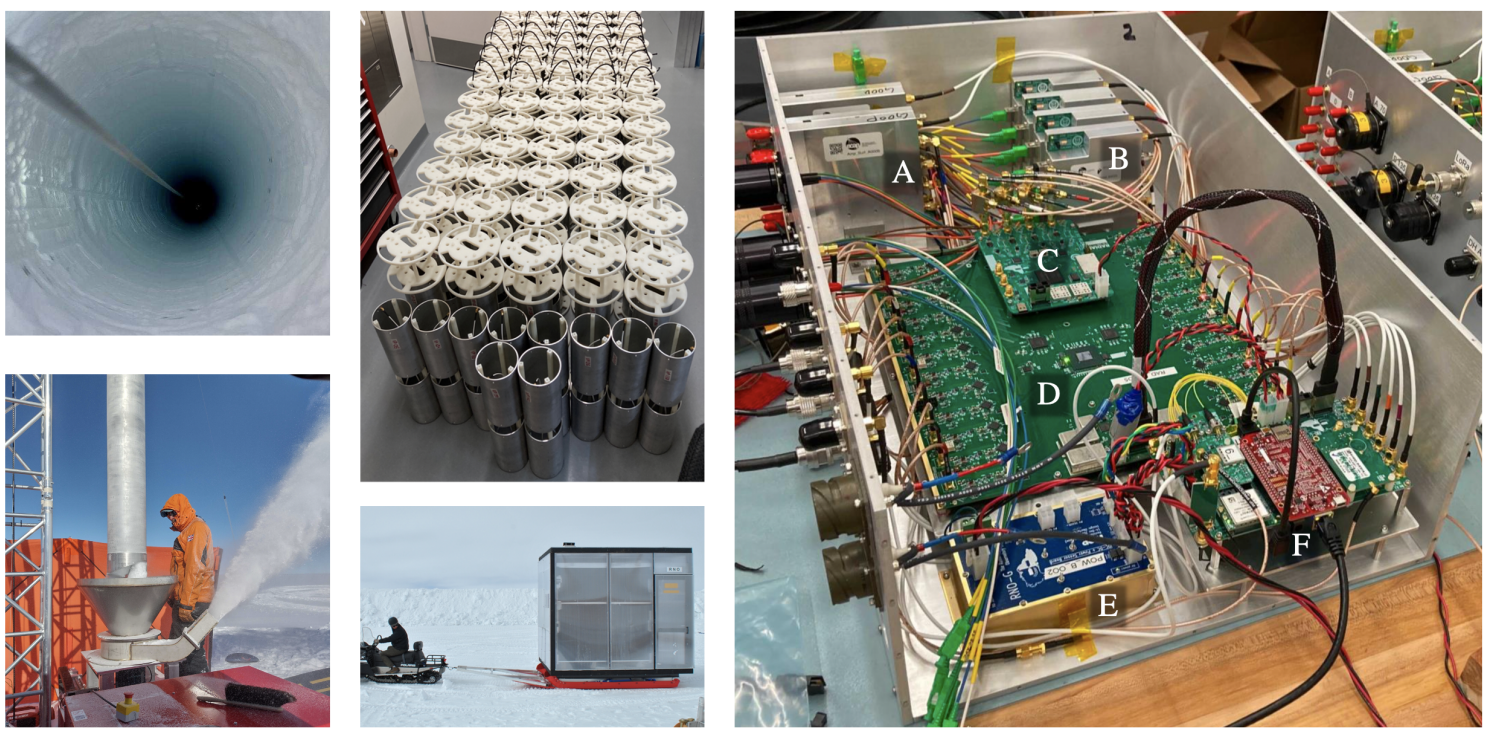

Figure 5: Pictures from construction and deployment of the first stations in the 2021 season.

Left, Top: Finished borehole. Left, Bottom: ASIG borehole drill in operation.

Middle, Top: All VPol antennas before deployment.

Middle, Bottom: Deployment shake being transported into position over borehole.

Right: Nearly complete DAQ box, with A surface amplifiers, B deep amplifiers and RFoF reciever, C low-threshold trigger, $\mathbf{D}$ digitizer, $\mathbf{E}$ power regulation board, $\mathbf{F}$ Controller board with single board computer, GPS unit and calibration pulser source daughter boards.

A horizontally polarized (Hpol) antenna inside of a borehole is a challenge to construct since the 11.2" diameter is much smaller than the wavelengths of interest, $\mathrm{O}(\mathrm{m})$. RNO-G iterated on the ARA collaboration design and designed a quadslot antenna, effectively a loop antenna with four feeds to improve azimuth symmetry and extended in the direction of the borehole to improve bandwidth. The four feeds are phased together and passed through a matching network to increase bandwidth. The antenna's usable band is $250-500 \mathrm{MHz}$ with a VSWR $<10$ in band. In-situ calibration is ongoing as well as studies of other Hpol designs, including a turnstile antenna.

\section{Deployment}

The first season of deployment started in May 2021 with the arrival of the drilling team and will continue until the last flight departs in August 2021. Deployment consists of three teams arriving at the station at different times. In order, they are the drilling (arrived in May), deployment (arrived in June) and calibration (arriving in July) teams, with deployment and drilling teams operating in tandem in June. To date, on-base infrastructure has been constructed (including the LTE network, LoRAWAN network, station data server and off-station telemetry), borehole drilling continues, and the first station has been deployed. We project the first five stations will be deployed by the end of the season in August 2021, a great accomplishment in the context of complications due to the ongoing COVID pandemic. 


\section{Conclusion}

The RNO-G experiment development and deployment is ongoing, with most systems coming online for the first time during the ICRC 2021 conference. We have developed hardware for a detector composed of modular, autonomous stations, including wireless communication, solar power system, low-threshold triggering and borehole-deployed antennas. RNO-G will be composed of 35 stations by 2024 and, after a five-year science run, the array will have world-leading sensitivity to the astrophysical neutrino flux at energies above $10 \mathrm{PeV}$.

\section{References}

[1] RNO-G Collaboration, J. A. Aguilar et al. JINST 16 no. 03, (2021) P03025.

[2] IceCube Collaboration, M. G. Aartsen et al. Science 342 (2013) 1242856.

[3] IceCube Collaboration, M. G. Aartsen et al. Science 361 no. 6398, (2018) 147-151.

[4] R. Stein et al. Nature Astron. 5 no. 5, (2021) 510-518.

[5] IceCube-Gen2 Collaboration, M. G. Aartsen et al. J. Phys. G 48 no. 6, (2021) 060501.

[6] J. M. Roberts, E. Oberla, P. Allison, G. S. Varner, S. Spack, B. Fox, and B. Rotter Nucl. Instrum. Meth. A 925 (2019) 92-100.

[7] P. Allison et al. Nucl. Instrum. Meth. A 930 (2019) 112-125.

[8] ARIANNA Collaboration, A. Anker et al. JINST 15 no. 09, (2020) P09039.

[9] S. W. Barwick et al. Astropart. Phys. 62 (2015) 139-151.

[10] C. Deaconu, A. G. Vieregg, S. A. Wissel, J. Bowen, S. Chipman, A. Gupta, C. Miki, R. J. Nichol, and D. Saltzberg Phys. Rev. D 98 no. 4, (2018) 043010.

[11] C. Glaser et al. Eur. Phys. J. C 80 no. 2, (2020) 77.

[12] I. Kravchenko, S. Hussain, D. Seckel, D. Besson, E. Fensholt, J. Ralston, J. Taylor, K. Ratzlaff, and R. Young Physical Review D 85 no. 6, (Mar, 2012). 


\section{Full Authors List: Collaboration}

J. A. Aguilar ${ }^{1}$, P. Allison ${ }^{2}$, J. J. Beatty ${ }^{2}$, H. Bernhoff ${ }^{3}$, D. Besson ${ }^{4,5}$, N. Bingefors ${ }^{6}$, O. Botner ${ }^{6}$, S. Bouma ${ }^{7}$, S. Buitink ${ }^{8}$, K. Carter ${ }^{9}$, M. Cataldo ${ }^{7}$, B. A. Clark ${ }^{10}$, Z. Curtis-Ginsberg ${ }^{11}$, A. Connolly ${ }^{2}$, P. Dasgupta ${ }^{1}$, S. de Kocker ${ }^{12}$, K. D. de Vries ${ }^{12}$, C. Deaconu ${ }^{11}$, M. A. DuVernois ${ }^{13}$, C. Glaser ${ }^{6}$, A. Hallgren ${ }^{6}$, S. Hallmann ${ }^{14}$, J. C. Hanson ${ }^{15}$, B. Hendricks ${ }^{16}$, B. Hokanson-Fasig ${ }^{13}$, C. Hornhuber ${ }^{4}$, K. Hughes ${ }^{11}$, A. Karle ${ }^{13}$, J. L. Kelley ${ }^{13}$, S. R. Klein ${ }^{17}$, R. Krebs ${ }^{16}$, R. Lahmann ${ }^{7}$, U. Latif ${ }^{12}$, M. Magnuson ${ }^{4}$, T. Meures ${ }^{13}$, Z. S. Meyers ${ }^{14,7}$, K. Mulrey ${ }^{8}$, A. Nelles ${ }^{14,7}$, A. Novikov ${ }^{4}$, E. Oberla ${ }^{11}$, B. Oeyen ${ }^{18}$, H. Pandya ${ }^{8}$, I. Plaisier ${ }^{7,14}$, L. Pyras ${ }^{14,7}$, D. Ryckbosch ${ }^{18}$, O. Scholten ${ }^{12,19}$, D. Seckel ${ }^{20}$, D. Smith ${ }^{11}$, D. Southall ${ }^{11}$, J. Torres ${ }^{2}$, S. Toscano ${ }^{1}$, D. Tosi ${ }^{13}$, D. J. Van Den Broeck ${ }^{12,8}$, N. van Eijndhoven ${ }^{12}$, A. G. Vieregg ${ }^{12}$, C. Welling ${ }^{7,14}$, S. Wissel ${ }^{16,9}$, R. Young ${ }^{4}$, and A. Zink ${ }^{7}$

${ }^{1}$ Université Libre de Bruxelles, Science Faculty CP230, B-1050 Brussels, Belgium

${ }^{2}$ Dept. of Physics, Center for Cosmology and AstroParticle Physics, Ohio State University, Columbus, OH 43210, USA

${ }^{3}$ Uppsala University, Dept. of Engineering Sciences, Division of Electricity, Uppsala, SE-752 37, Sweden

${ }^{4}$ University of Kansas, Dept. of Physics and Astronomy, Lawrence, KS 66045, USA

${ }^{5}$ National Nuclear Research University MEPhI, Kashirskoe Shosse 31, 115409, Moscow, Russia

${ }^{6}$ Uppsala University, Dept. of Physics and Astronomy, Uppsala, SE-752 37, Sweden

${ }^{7}$ Erlangen Center for Astroparticle Physics (ECAP), Friedrich-Alexander-University Erlangen-Nuremberg, 91058 Erlangen, Germany

${ }^{8}$ Vrije Universiteit Brussel, Astrophysical Institute, Pleinlaan 2, 1050 Brussels, Belgium

${ }^{9}$ Physics Dept. California Polytechnic State University, San Luis Obispo CA 93407, USA

${ }^{10}$ Dept. of Physics and Astronomy, Michigan State University, East Lansing MI 48824, USA

${ }^{11}$ Dept. of Physics, Enrico Fermi Inst., Kavli Inst. for Cosmological Physics, University of Chicago, Chicago, IL 60637, USA

${ }^{12}$ Vrije Universiteit Brussel, Dienst ELEM, B-1050 Brussels, Belgium

${ }^{13}$ Wisconsin IceCube Particle Astrophysics Center (WIPAC) and Dept. of Physics, University of Wisconsin-Madison, Madison, WI 53703, USA

${ }^{14}$ DESY, Platanenallee 6, 15738 Zeuthen, Germany

${ }^{15}$ Whittier College, Whittier, CA 90602, USA

${ }^{16}$ Dept. of Physics, Dept. of Astronomy \& Astrophysics, Penn State University, University Park, PA 16801, USA

${ }^{17}$ Lawrence Berkeley National Laboratory, Berkeley, CA 94720, USA

${ }^{18}$ Ghent University, Dept. of Physics and Astronomy, B-9000 Gent, Belgium

${ }^{19}$ Kapteijn Institute, University of Groningen, Groningen, The Netherlands

${ }^{20}$ Dept. of Physics and Astronomy, University of Delaware, Newark, DE 19716, USA 